\title{
Multi-Provider Cloud Computing Network Infrastructure Optimization
}

\author{
Thepparit Banditwattanawong ${ }^{1, a}$, Masawee Masdisornchote ${ }^{1, a, *}$, Putchong \\ Uthayopas $^{\mathrm{b}}$ \\ ${ }^{a}$ School of Information Technology, Sripatum University, Bangkok, Thailand. \\ ${ }^{b}$ Computer Engineering Department, Kasetsart University, Bangkok, Thailand.
}

\begin{abstract}
Cloud-adopting enterprises have been increasingly employing multiple cloud providers concurrently, for example, to consume unique services and to mitigate data lock-in risk. As a consequence, the enterprises must be able to address contrasting quality-of-service degrees offered by the different providers. This paper presents an intelligent cloud cache eviction approach, namely iCloud, as the core component of a client-side cloud cache. i-Cloud is capable of reducing public cloud data-out expenses, improving cloud network scalability and lowering cloud service access latencies specifically in multi-provider cloud environments. Trace-driven simulations have showed that i-Cloud outperformed well-known approaches in all performance metrics. In addition, i-Cloud is not only able to achieve optimal performances in all metrics simultaneously but also delivered relatively stable performances across all performance metrics. The results have also indicated that taking the nonuniformity of data-out charge rates into cache eviction decisions improved caching performances in all metrics.
\end{abstract}

Keywords: Cache replacement, Multi-provider cloud, Federated cloud, Hybrid cloud, Artificial neural network, Cost-saving ratio

\footnotetext{
${ }^{*}$ Corresponding author.

Email address: masawee.ma@spu.ac.th (Masawee Masdisornchote)

${ }^{1}$ Sripatum university, 61 Phaholyothin Road, Jatujak, Bangkok 10900, Thailand.
} 


\section{Introduction}

Big data has been increasingly hosted in clouds as in, for example, Facebook, Youtube, Street View Google Maps, iTune Store. This is because cloud enables not only fast, economical and greener big data analytics $[1,2,3]$ but also highly-scalable and distributed sharing of the data. This is the evolution of data volume, data placement and data consumption behavior that altogether has led both practitioners and researchers to address several new cloud-computing problems including the downstream bandwidth saturation of network connections between external cloud and consumer premises, increase in external private-cloud data-out charge imposed by public cloud providers (e.g., [4], [5] and [6]) and long-delayed cloud service responsiveness. These difficulties have been recognized in $[7,8,9,10]$ as the forms of data transfer bottlenecks, data transfer costs or cloud computing economics and SaaS SLA responsiveness. A straightforward way to handle these problems is network bandwidth upgrade, which unfortunately impedes cloud economy. A wiser means is disk shipping by overnight delivery services [7, 8] that is only applicable for delay-tolerant cloud services. Another solution to meet these scalability, economy and responsiveness requirements of cloud computing services at the same time is the consumer-initiated partial replication of cloud-hosted data to consumer (nearby) locus as in Amazon CloudFront [11], which offers a caching service through content delivery network. We refer to this solution as client-side cloud caching.

Client-side cloud caches are located in or nearby consumer premises where HTTP requests to external private clouds are proxied by the cloud caches, which in turn reply with the valid copies of the requested data objects either from their local storages (i.e., cache hits) or by downloading updated copies from the clouds (i.e., cache misses). The cloud caches are basically evolved from traditional forward web caching proxies since cloud data is also delivered by using the same set of HTTP/TCP/IP protocol stacks as in WWW. Unavoidably, the same limitation as in web caching is also shared in the cloud caches that is caching entire remote data in local cache storage is not economically sensible, thus a cache replacement policy is also mandatory for the cloud caches. In fact, client-side cloud caches can also be deployed in a hierarchical structure as portrayed in Fig. 1: the web-browser level cloud caches are nonshared by individuals whereas the enterprise- and ISP-level cloud caches are shared. This paper focuses on a client-side shared cloud

cache at the enterprise level. Another main characteristic of the client-side 


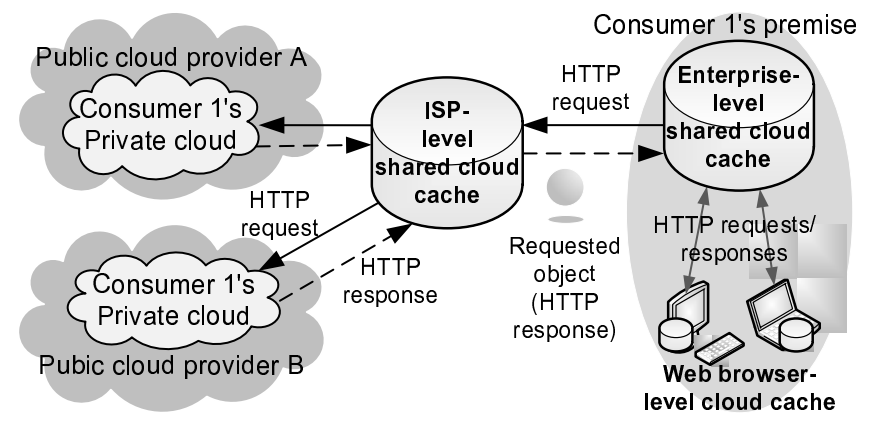

Figure 1: Cloud cache deployment scenario in a multi-provider cloud environment.

shared cloud cache is multi-tenancy support whereby workloads initiated by different enterprises are shared for hardware but isolated for user data. The other properties of the client-side shared cloud cache lie in its various Cacheas-a-Service (CaaS) models [12] (e.g., RAM Multitenancy Isolated database, and SSD Multitenancy Isolated database), which are not offered by the traditional web caching proxies.

As illustrated in Fig. 1, an enterprise-level cloud cache can be deployed in a multi-provider cloud environment where a consumer enterprise employs multiple concurrent cloud providers to host its external private clouds that are either independent from each other $[13,14]$ or interconnected as a hybrid cloud [15]; both are the forms of federated cloud [16, 17]. There are several benefits of multi-provider cloud deployment such as load balancing [18, 19], enabling of planned downtime for system maintenance [17], confidentiality protection [20], risk mitigation (mandated by hospitals, stock markets, air transportation controls, etc.) $[20,21]$ and the utilization of unique capabilities offered by different cloud providers [13]. Nevertheless, the deployment of multiple cloud providers usually imposes different levels of QoS perceived by consumers in the forms of unbalancing downstream network throughputs and nonuniform cloud data-out charge rates. This complicates cloud caching optimization in that cloud cache replacement must be aware of QoS heterogeneity to achieve both efficiency and economy. To accomplish such optimization, our extensive investigation of related work in Section 6 gives several reasons that we cannot simply exploit existing web cache eviction techniques. The other reason for that client-side cloud cache replacement policies must be designed differently from the traditional WWW ones lies in 
the intrinsic characteristic of cloud computing data itself: Cloud data objects (e.g., big data) have larger sizes than traditional WWW objects [22]. To our current experiences, requesting for big objects made available via cloud computing services (e.g., cloud storages) still keep users waiting for long whereas downloading small objects from clouds nowadays is fast as if the objects were fetched from user locality. This means that priority must be given to the loading optimization of big objects whereas existing web cache replacement policies had been totally optimized for small objects [23, 24] (because, in the past, fetching small data object was so delayed due to slow Internet connection that it was unacceptable for users to experience such delays on every request).

As an early attempt in the field of enterprise-level client-side shared cloud caching towards multi-provider clouds, this paper presents an intelligent cloud cache replacement policy, i-Cloud (named so for its intended application domain), along with its technical and economical performances and important findings.

The organization of this paper is as follows. Section 2 explains monetary cost models as a basis for multi-provider cloud employment. Section 3 explains our experimental data sets and how the raw traces were preprocessed to obtain training and evaluation data sets that realistically represent the traffics of shared cloud networks. i-Cloud is described in details in Section 4. Section 5 discusses comparative performance results and presents algorithmic success factor analysis to formulate an open question for future research in this new field of study. We extensively contrast and compare this work with relevant ones in Section 6. Section 7 draws the conclusion of crucial findings.

\section{Monetary Cost Models}

When an organization employs cloud computing, there are two possible monetary cost models [25] depending on the number of contracted cloud providers. If a single provider is contracted, a uniform cost model is employed in which object requests made by the organization entirely go to the same cloud provider, thus a single data-out charge rate is applied to all of the requested objects. Otherwise, the other nonuniform cost model is applied in which object requests separately go to multiple cloud providers, offering different data-out charge rates. In other words, objects retrieved from the same cloud service domain were always charged by the same provider. 
Since this paper aims for multi-provider clouds, both i-Cloud training (Section 4.2.2) and i-Cloud evaluation (Section 5) are based on the nonuniform cost model. In particular, we assumed that an organization's cloudhosting domains were hosted separately by two contracted public cloud providers, who offer different data-out charge rates, thus a pair of monetary costs associated with data-out transfers. The first provider was Google and thus its charge rate was set to 0.1535 USD/GB while the other provider was AWS and its charge rate was set to 0.0829 USD/GB.

Both of the flat charge rates were converted from their original regressive rates (i.e., Google Cloud Storage's network egress charge in Asia-Pacific region as of September 2013 [5] and Amazon S3's data transfer out to Internet charge (US Standard) as of September 2013 [4]) by means of weighted averages: First, we determined the total amount of data transferred out of each provider to which a regressive rate was applied. Once, we knew the total data-out volume and the range of corresponding regressive rates, we can figure out the total expense and consequently the flat charge rate in USD/GB for such provider. The total data-out volume was realistically assumed based on a representative scenario where an organization utilizing data residing in cloud by transferring it out of the cloud through 10 Gbps Metro Ethernet with $50 \%$ average downstream bandwidth utilization for 8 work hours a day requires the total amount of cloud data-out transfer 4,570.31 TB per year (as of 260 workdays per year) or 380.86 TB per month; we also assumed for the ease of understanding that the workload 380.86 TB per month is distributed equally among the two public cloud providers to emulate a multi-provider cloud circumstance, thus a data-out volume is $190.43 \mathrm{~TB}$ per provider-month. This split volume costs 29,925.88 USD/month for one provider and 14,704.74 USD/month for the other provider making the organization liable to pay for cloud data-out transfer totally 44,630.62 USD/month.

\section{Experimental Data Sets}

To understand the following sections, it is necessary to clarify our original data sets and their decendant ones used throughout this study.

Since a cloud consumer enterprise usually deploys various cloud computing services and locate them by specific domain names. This means that an enterprise-level shared cloud cache (the focus of this paper) must serve mixed HTTP request streams to multiple cloud services. Hence, raw HTTP traces, each of which was composed of mixed request streams generated by a 
Table 1: Cloudiness characteristics of the preprocessed traces.

\begin{tabular}{ccc}
\hline Feature & 31-day BO trace & 31-day NY trace \\
\hline Total cloud-hosted domains & $40(80 \%)$ & $35(70 \%)$ \\
Total cloud service references & $519,016(81.2 \%)$ & $1,001,821(76.4 \%)$ \\
Total unique cloud-data objects & $296,248(91.4 \%)$ & $440,324(74.2 \%)$ \\
Maximum bytes of total & $3,277,620,746(79 \%)$ & $8,848,209,001(51.8 \%)$ \\
requested cloud-data objects & & \\
\hline
\end{tabular}

single user community (emulating the cloud consumer enterprise), were used as our original traces. In particular, we utilized a pair of traces [26] of different user communities to cross-check our results and findings: a 31-day BO trace was gathered from a user community in Boulder from 16th August to 15th September 2012, and a 31-day NY trace was collected from the other user community in New York from 16th July to 15th August 2012. Since these traces not only contain references to the domain names of presently well-known cloud computing services, such as Youtube and Facebook, but also their major parts represent cloud traffics (cf. Table 1), we believe that they are still valid at the time this paper is in a publication process

However, to emulate the cloud service request stream of a single consumer organization in a more realistic way, each of the original traces must be preprocessed to reduce the number of domain names referenced inside the trace. Omitting this stage, otherwise, misleadingly means that the organization owns an impractically large number of cloud services (as logged within each single trace) hosted totally in its own private cloud. We thus extracted the object references of merely 50 (most popular) domain names (to emulate the whole number of cloud service domains administrated within the first author's university).

The extracted references that form each preprocessed trace reflected not only WWW requests but also cloud service requests to, for instance, Facebook, Youtube, Twitter, Google SaaSes, and Mediafire as well as 4shared IaaSes. Table 1 quantitatively characterized cloud service references contained within each of the preprocessed traces: Total cloud-hosted domain names express the total number of domain names identified as cloud services out of the entire 50 domains; total cloud service references are the total number of data requests to the identified cloud domain names; total unique cloud-data objects denote the total number of unique data objects requested 
from the identified cloud domain names; maximum bytes of total requested cloud-data objects indicate the maximum volume of data requested from the cloud services; the parenthesized values (referred to as cloudiness) show the features in proportion to each complete trace. We could see that it was the cloud traffic instead of the WWW one that took majority part in both traces. In fact, the WWW references contained in the preprocessed traces could represent either traditional WWW data requests (i.e., requests to oldstyle web hosts) or cloud data requests (i.e., requests to cloud-hosted web servers). In the absence of reliable information for identifying the WWW references' server hosting infrastructure types (which are either cloud or traditional infrastructures), we assumed that these WWW references entirely represent the requests to cloud-hosted web servers, thus regarded as cloud data requests as well. We believed that this assumption was realistic as several present application service providers have been offering cloud-based web hosting services rather than old-style web hosting or co-location services (i.e., the WWW references have been increasingly going to clouds).

To implement the nonuniform cost model, each of the preprocessed traces would be used in such a way that its entire object references were assumed to be made by a single organization and distributedly go to two external private clouds hosted separately by the two public cloud providers introduced in Section 2. Therefore, each preprocessed trace contained two separate object reference streams, each of which was served by the different provider. To have the object reference streams within each of the trace charged at different data-out charge rates while references in the same stream are charged at the same rate (as processed by the same public cloud provider), we divided the 50 domains randomly into two equal groups of 25 domains each then associating each of the charge rates (0.1535 USD/GB and 0.0829 USD/GB figured out in Section 2) with each entire stream.

As for the other preprocessing tasks, all references representing dynamic object requests were not excluded from the traces to reflect actual caching performances against all types of requests. (That is why our absolute performance results were not so high as those reported in some of the related works.) The final steps preprocessing was the removals of unused fields and appending object expiration times as a new field to all object references. The expiration times were figured out based on the following heuristic rules, which conform to the definition of resource age in [27], with an assumption that object creators set object expiration times deliberately. 
Table 2: Characteristics of all experimental data sets.

\begin{tabular}{ccccc}
\hline \multirow{2}{*}{ Feature } & \multicolumn{2}{c}{ BO } & \multicolumn{2}{c}{ NY } \\
\cline { 2 - 5 } & 15 days & 31 days & 15 days & 31 days \\
\hline Total requests & 352,224 & 639,187 & 639,199 & $1,311,880$ \\
\hline Total requested bytes & $2,294,688,191$ & $4,149,211,314$ & $6,499,655,874$ & $17,067,821,671$ \\
\hline Total unique objects & 181,624 & 323,979 & 290,851 & 593,365 \\
\hline $\begin{array}{c}\text { Maximum bytes of } \\
\text { total unique objects }\end{array}$ & $1,386,970,321$ & $2,262,144,480$ & $4,791,008,825$ & $10,801,010,237$ \\
\hline
\end{tabular}

- Rule 1: By scanning down all references within a trace in a timestamp order, an object expired immediately after its size was found changed from its last reference.

- Rule 2: As long as the size of object remained unchanged, its lifetime was extended to its last request of such an object size as appeared in a trace.

- Rule 3: An object apparent only once throughout a trace expired suddenly after its use. (This object tends to be a kind of dynamic one, which is by default not cached by Squid. Following this Squid's recommendation, this rule is prescribed so.)

At this point, the one-month $\mathrm{BO}$ and NY traces were preprocessed completely but we also created two more shorter traces from the preprocessed traces: the references belonging to the first 15 days of each one-month trace were duplicated into a new trace resulting in a 15-day BO trace and a 15day NY trace. This allowed objects referenced in both 15-day traces to have maximum one-month lifespans (as a result of applying the above heuristic rules to the one-month traces before the duplications) rather than merely 15 days, and avoided false positives for static objects made by the heuristic rule 3. All of four finally preprocessed traces are characterized in Table 2. Notice that the last feature must be denoted as the maximum bytes (of total unique objects) since the sizes of several unique objects changed over a reference stream as detected in each trace. 


\section{A Proposed Cloud Cache Replacement Policy}

To design a cloud cache eviction approach requires the new perspective of problem understanding as justified in Section 1. A set of the design goals of i-Cloud that truly accommodates the efficient decisions of cached object evictions in cloud computing environments are the optimization of cost-saving ratio, byte-hit ratio and delay-saving ratio. These three performance metrics including a standard hit rate metric are revisited below. The detailed statements of the design goals have been presented in [28]. For all objects $1 \leq i$ $\leq \mathrm{N}$

$$
\begin{gathered}
\text { byte-hit ratio }=\frac{\sum_{i=1}^{N} s_{i} h_{i}}{\sum_{i=1}^{N} s_{i} r_{i}} \\
\text { cost-saving ratio }=\frac{\sum_{i=1}^{N} c_{i} s_{i} h_{i}}{\sum_{i=1}^{N} c_{i} s_{i} r_{i}} \\
\text { delay-saving ratio }=\frac{\sum_{i=1}^{N} l_{i} h_{i}}{\sum_{i=1}^{N} l_{i} r_{i}} \\
\text { hit rate }=\frac{\sum_{i=1}^{N} h_{i}}{\sum_{i=1}^{N} r_{i}}
\end{gathered}
$$

where $\mathrm{N}$ is the total number of cached objects, $s_{i}$ is the size of $i, h_{i}$ is how many times a valid copy of $i$ is fetched from cache, $r_{i}$ is the total number of requests to $i, l_{i}$ is the downloading latency of $i$ from cloud, and $c_{i}$ is the data-out charge rate or monetary cost per object size for downloading $i$ from cloud. The cost-saving metric has been originally proposed in [29]. It is particularly useful for demonstrating economical performance in multiprovider cloud employment. Since economy is highlighted as the key benefit of cloud computing, any future strategies are encouraged to use this metric.

Fig. 2 demonstrates the conceptual framework of i-Cloud. It consists of two main processing modules, Cloud cache replacement policy and a forecaster. We explain these modules in two following subsections.

\subsection{Cloud cache replacement policy module}

This module implemented our previous work called Cloud cache replacement policy (cf. the end of Section 6). Table 3 presents the pseudo code of Cloud algorithm, which can be explained as follows. When cache eviction 


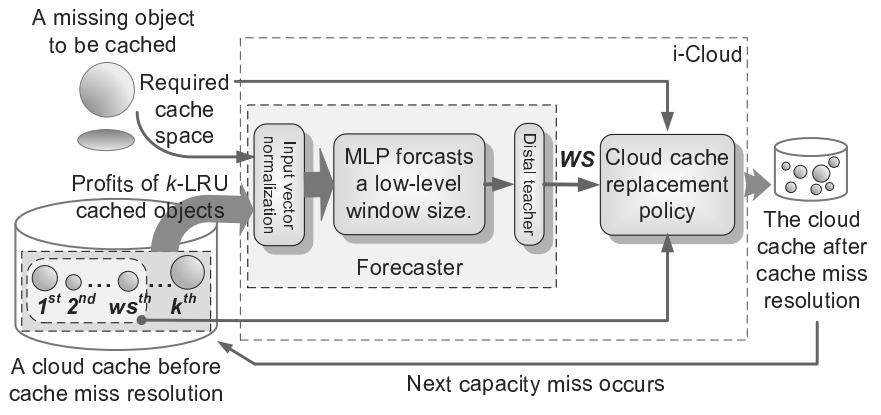

Figure 2: Conceptual framework of i-Cloud cache replacement policy.

is needed and Cloud cache replacement mechanism is invoked, it first formulates a cluster of in-cache LRU objects as many as instructed either by a window size parameter or a required cache space (which is equal to at least a requested missing object's size and can be fuzzy as in Squid using low and high watermarks for object eviction controls [30]) depending on which one is larger: if the number of objects specified by window size has bigger total object size than the required cache space then the window size will be used. Once the cluster of LRU objects has been formed, Cloud quantifies a profit associated with each object inside the cluster by using Eq.(5) whose design rationale can be found in [28]. Given an object $i$,

$$
\text { profit }_{i}=s_{i} c_{i} l_{i} f_{i} T T L_{i}
$$

where $s_{i}$ is the size (in bytes) of $i, c_{i}$ is data-out charge rate (i.e., monetary cost per object size) for downloading $i, l_{i}$ is the latency (in milliseconds) of downloading $i, f_{i}$ is the access frequency of $i$ (i.e., the number of requests to $i$ since it has been cached lastly), and $T T L_{i}$ is the remaining lifespan (in milliseconds) of $i$. (In fact, we have tried a number of parameter weighting possibilities to tune the combinative value of these parameters and finally come out at this simplest and most efficient profit function.) An object with least profit value is evicted first from a cache. This object eviction process is repeated on the next least profitable objects in the cluster until gaining enough cache room that contains the required cache space. The parameter $c_{i}$ enables i-Cloud to support the nonuniform cost model introduced in Section 2. In the uniform cost model, the value of $c_{i}$ in Eq.(2) is identical for all objects and thus can be eliminated. This means that cost-saving ratio is absolutely equal to byte-hit ratio in the uniform cost model. In practice, 
Table 3: Cloud algorithm

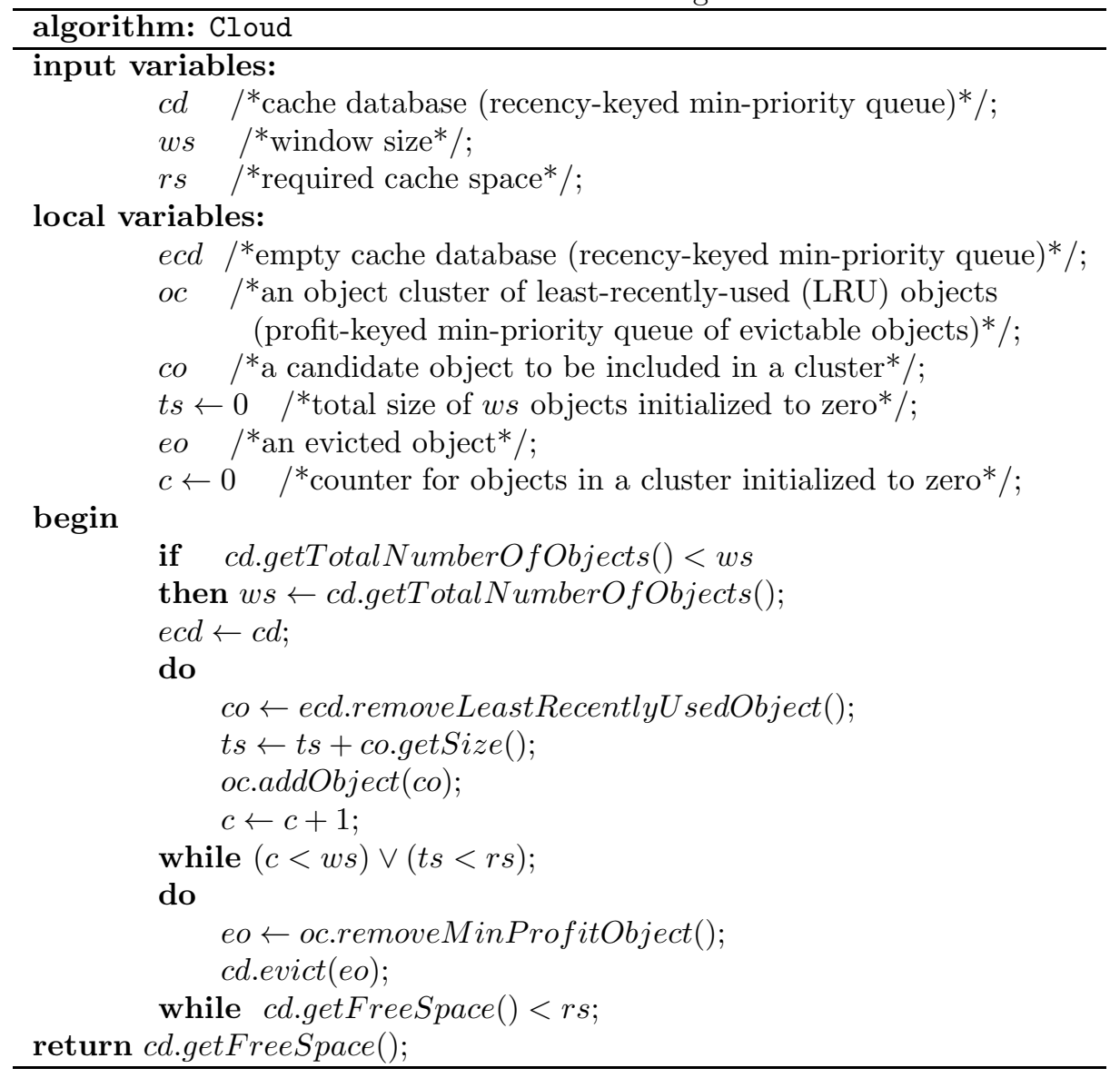

the TTL value can be derived from the Expires or the Max-age field inside HTTP protocol header [31]. The other parameters in Eq.5, $s_{i}$ and $l_{i}$, can be supplied directly by means of runtime measurements, $c_{i}$ can be preconfigured by i-Cloud administrators, and $f_{i}$ and access recency can be cumulatively figured out at runtime.

A parameter influencing all performance aspects of i-Cloud is window size (input variable $w s$ in Table 3 ). We have determined both optimal window sizes $\left(w s_{o}\right)$ and worst window sizes $\left(w s_{w}\right)$ by extensively trial-and-error experiments as shown in Table 4. In particular, each optimal window size had been tuned towards a maximum cost-saving ratio for a certain data set and cache size based on the nonuniform cost model. Since several different window sizes 
Table 4: Window-size parameter values based on nonuniform cost models.

\begin{tabular}{ccccccccc}
\hline & \multicolumn{4}{c}{ BO } & \multicolumn{5}{c}{$\mathrm{NY}$} \\
\cline { 2 - 9 } Cache size $(\%)$ & \multicolumn{2}{c}{15 days } & \multicolumn{2}{c}{31 days } & \multicolumn{1}{c}{15 days } & \multicolumn{2}{c}{31 days } \\
\cline { 2 - 9 } & $w s_{o}$ & $w s_{w}$ & $w s_{o}$ & $w s_{w}$ & $w s_{O}$ & $w s_{w}$ & $w s_{o}$ & $w s_{w}$ \\
\hline 10 & 900 & 10,000 & 700 & 9,700 & 200 & 9,900 & 4,800 & 100 \\
\hline 20 & 1,300 & 9,400 & 200 & 100 & 8,800 & 100 & 3,400 & 100 \\
\hline 30 & 200 & 100 & 8,400 & 100 & 400 & 100 & 3,900 & 200 \\
\hline
\end{tabular}

were found to yield identical maximum byte hit, the smallest window size was selected as an optimal one due to requiring less processing time. The optimal window sizes yielded cost-saving performance maxima, whereas the worst window sizes produced cost-saving performance minima as they had been tuned towards minimum cost-saving ratios using specific data sets and cache sizes based on the nonuniform cost model. In Table 4, all cache sizes are presented in the percent of the maximum bytes of total unique objects of the one-month data sets in Table 2.

\subsection{Forecaster module}

To deploy i-Cloud, the effective method of window size selection is needed to forecast optimal window sizes and avoid the worst ones for a given workload as aforementioned. As we can see from Table 4 that the optimal window sizes varied from one input data set and cache size to another. Therefore, i-Cloud must be able to adapt its window size value properly to an evolving workload. This requirement is actually a kind of function approximation problem: given an input pattern $x$ and a corresponding target output $t$, a goal is to find an unknown function $f$ that matches the target output for each input pattern that is $t=f(x)$. We have thus proposed i-Cloud based on the cooperation of Cloud policy and an artificial neural network, in particular, a multilayer feedforward neural network or a multilayer perceptron (MLP) as shown in Fig. 2. MLPs have long been accepted to be powerful enough to serve as universal approximators for implementing essentially any functions [32]. With an MLP, i-Cloud is so intelligent that it automatically forecasts near-optimal window sizes in an automated real-time manner.

The forecaster module in Fig. 2 operates as follows. When a capacity miss takes place (i.e., a request for an object that was in a cache but has been since purged, thus cache eviction is required to serve the request [33]), the vector of $k$ LRU cached objects' profits together with a required cache 
space are fed into the forecaster to forecast a near-optimal window size. During this stage, each input vector is passed internally into the input vector normalization process then the normalized vector is presented to the MLP component to forecast a low-level window size. Such a low-level window size is later denormalized by a distal teacher component (responsible for transforming actions produced by the MLP into environmental outcomes [34]) to obtain an actual (potentially near-optimal) window size. The actual window size is subsequently presented to Cloud cache replacement module, which follows Cloud algorithm as described earlier.

To construct the forecaster module mandates the knowledges of the MLP's structure, an input vector normalization method, a distal teacher function, and some MLP training and validation configurations as follows.

\subsubsection{MLP structure}

MLP structure prescribes the number of layers and nodes to be placed in each layer of the MLP. Our goal is to find the smallest possible structure since too large MLP learns a training set well but is unable to generalize [32]. We have designed the MLP structure based on the following guidelines.

- The appropriate number of input nodes were determined by two lessons learned from our previous studies [25, 28]. First, both current cache state, which is influenced by preceding object requests (i.e., there existed correlations in each request stream) together with a total allocated cache size and a required cache space were main factors to window size optimization. This design guideline has been implemented as shown in Fig. 2: a current cache state is captured in the form of a profit vector while a missing object's size is used to indicate a minimum required cache space. Second, the optimal window sizes ranged between 100 and 8,000 meaning that the lowest profitable objects to be purged from cache were potentially found inside a cluster of 8,000 LRU cached objects. Leveraging this fact simplifies and practicalizes the MLP's implementation since a current cache state can be snapshot by examining the part of cache database rather than the whole one, which can be much more time consuming. However, to give chance for any unveiled maximum values of optimal window sizes, we have chosen 10,000 (i.e., $k$ in Fig. 2) LRU cached objects to represent each current cache state to be examined by the MLP. Thus, the total number of input nodes is 10,002 including a required cache space node and a bias one. 


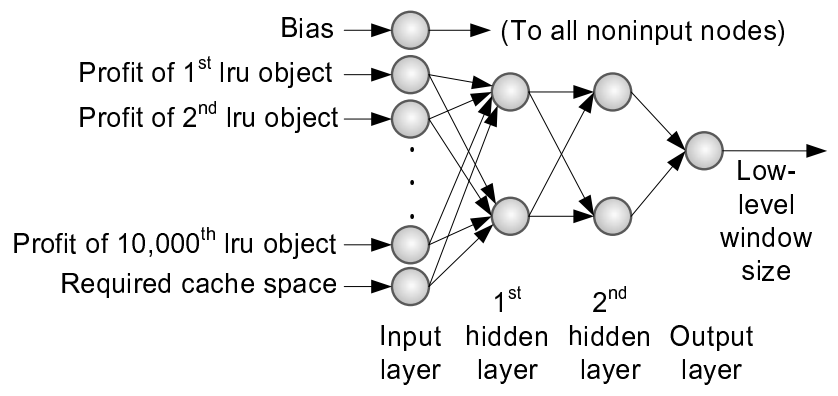

Figure 3: The MLP structure of i-Cloud's forecaster.

- It is guided in [32] that some continuous functions cannot be approximated accurately by single-hidden-layer MLP whereas two hidden layers are sufficient to approximate any desired bounded continuous function, which is also the case of i-Cloud. Moreover, the hidden layers are usually kept at approximately the same size to ease training.

- The output layer necessitates only a single node to deliver an estimated low-level window size.

Several MLP structures were experimented in an effort based on these guidelines. We finally came out at a minimal structure that can be expressed in the conventional notation of $10,002 / 2 / 2 / 1$, which means 10,002 input nodes, two hidden layers with two nodes each, and a single output node as illustrated by Fig. 3. All the nodes are fully-connected between two adjacent layers, except a bias node is connected to all noninput ones. A complete input vector presented to the MLP is denoted as

$$
X=<1, p_{1}, p_{2}, \ldots, p_{10,000}, r s>
$$

where $p_{i=1}$ to 10,000 is the profit of the $i^{\text {th }}$ LRU object and $r s$ is a required cache space. Notice that the bias is set to a constant activation 1 .

\subsubsection{Learning phase}

Besides the MLP structure, to obtain the complete MLP mandates the appropriate set of weights to be associated with all links between the MLP's internal nodes. This has been done by means of supervised learning with a distal teacher and back-propagation: Training an MLP to learn relationship between training input patterns and a corresponding target output through 
Table 5: Characteristics of nonuniform cost-based training data sets.

\begin{tabular}{cccc}
\hline $\begin{array}{c}\text { Training } \\
\text { data set }\end{array}$ & $\begin{array}{c}\text { Size } \\
\text { (patterns) }\end{array}$ & $\begin{array}{c}\text { Original data set } \\
\text { and cache size }\end{array}$ & $\begin{array}{c}\text { Target } \\
\text { window size }\end{array}$ \\
\hline BO15D10\% & 55,169 & 15-day BO 10\% & 900 \\
NY15D10\% & 59,158 & 15-day NY 10\% & 4,800 \\
\hline
\end{tabular}

repetitive process guided by a back-propagation algorithm; during training, each input pattern is presented and forward-propagated through the MLP to produce an output; the output acts as an input to a distal teacher system, which transforms the output into a final one; difference between actual final and target outputs is an error measured by an objective function; the backpropagation algorithm then calculates the derivatives of the training errors and uses them to adjust the weights of the MLP to minimize the errors; the overall goal of the training is to minimize a total error for the given training patterns.

Our MLP learned input patterns inherent in caching state history. Each input pattern represented a cache miss state and was organized into a training vector of the form $X$ (Eq.(6)) and generated every time a capacity miss occurred during the i-Cloud simulation of a certain 15-day data set, a certain cache size and a respective optimal window size. The result of each complete simulation was a sequence of input patterns, which were contained inside a single training data set. By this means of training data set generation, we produced totally three training data sets based on $10 \%, 20 \%$ and $30 \%$ cache sizes per user-community data set presented in Table 2 by using the corresponding optimal window sizes in Table 4, then only the largest data set of each community was really utilized in the MLP's pattern learning process. Therefore, we prepared totally two different nonuniform cost-based training data sets shown in Table 5. If the network has $W$ adjustable weights, it is necessary to have on the order of $W / \epsilon$ patterns if an error rate $\epsilon$ is desired so that at least a fraction $1-\epsilon / 2$ are correctly classified [32]. Thus, both training data sets had adequate patterns to give low error rates 0.34 for NY15D10\% and 0.36 for BO15D10\%. As shown in the table, patterns within the same training data set were associated with a single target window size (i.e., a respective optimal window size in Table 4) because i-Cloud was found to give a successful performance results when using a fixed optimal window size per data set (as substantiated by [25] and [28]).

The input vector normalization and distal teacher components portrayed 
in Fig. 2 also contributed to the successful pattern learnings of the MLPs. Normalizing inputs into a certain range made the MLP dealing better with them [35] because a sigmoid output range was utilized more evenly. Input normalization also prohibited a sigmoid saturation problem that would cause slow training [32]. Therefore, we normalized every element value (except the bias constant) within every input vector by using max-min linear scaling: $v^{\prime}=v-\min \left(\max _{n e w}-\min _{n e w}\right) /(\max -\min )+\min _{\text {new }}$ where $v^{\prime}$ is the normalized value of a range [-1, 1], $v$ is an actual value, min is an actual minimal value found to be $0, \max$ is an actual maximum value observed throughout all of the training data sets to be approximately $10^{14}$, max $_{\text {new }}$ is a maximum normalized value 1 , and $\min _{\text {new }}$ is a minimum normalized value -1 . Therefore, we derived the following formula for the input vector normalization.

$$
v^{\prime}=2 v / 10^{14}-1
$$

where $v^{\prime}$ is the normalized value of a range $[-1,1]$ and $v$ is an actual value.

Because the MLP outputted a low-level window size of $[0.0,1.0]$ via sigmoid function, to achieve desired window sizes requires the distal teacher to denormalize the low-level window size. A distal teacher function used was linear scaling:

$$
y^{\prime}=10000 y
$$

where $y$ is an MLP output, 10000 is a maximum optimal window size (justified in Section 4.2.1), and $y^{\prime}$ is a denormalized output, which is an actual window size of $[0,10000]$.

Fig. 4 illustrates all steps of the MLP learning phase, which relied on a batch-mode back-propagation method. Among the steps, there were two major steps, forward propagation and back-propagation, explained in details as follows. During the forward propagation, every link in the MLP connecting node $j$ to node $i$ has an associated initial weight $w_{i j}$. Let $x_{j}$ denote the output activation of node $j$. Node $i$ computes a weighted sum $u_{i}$ of its inputs by $u_{i}=\sum_{j} w_{i j} x_{j}$. Index $j$ runs over all the indices $j<i$ of nodes in a lower layer than node $i$, including a bias node that can send input to node $i$. Not only $u_{i}$ but also external MLP input are passed through an activation function $f$ to obtain a node output activation $x_{i}=f\left(u_{i}\right)$. The activation function used here is a sigmoid function $f(x)=1 /\left(1+e^{-x}\right)$, which is the continuous, bounded, nondecreasing, nonlinear function of its input. It saturates at either 0 for large negative inputs or 1 for large positive inputs. During the backpropagation, the weights were adjusted through several training epoches by 


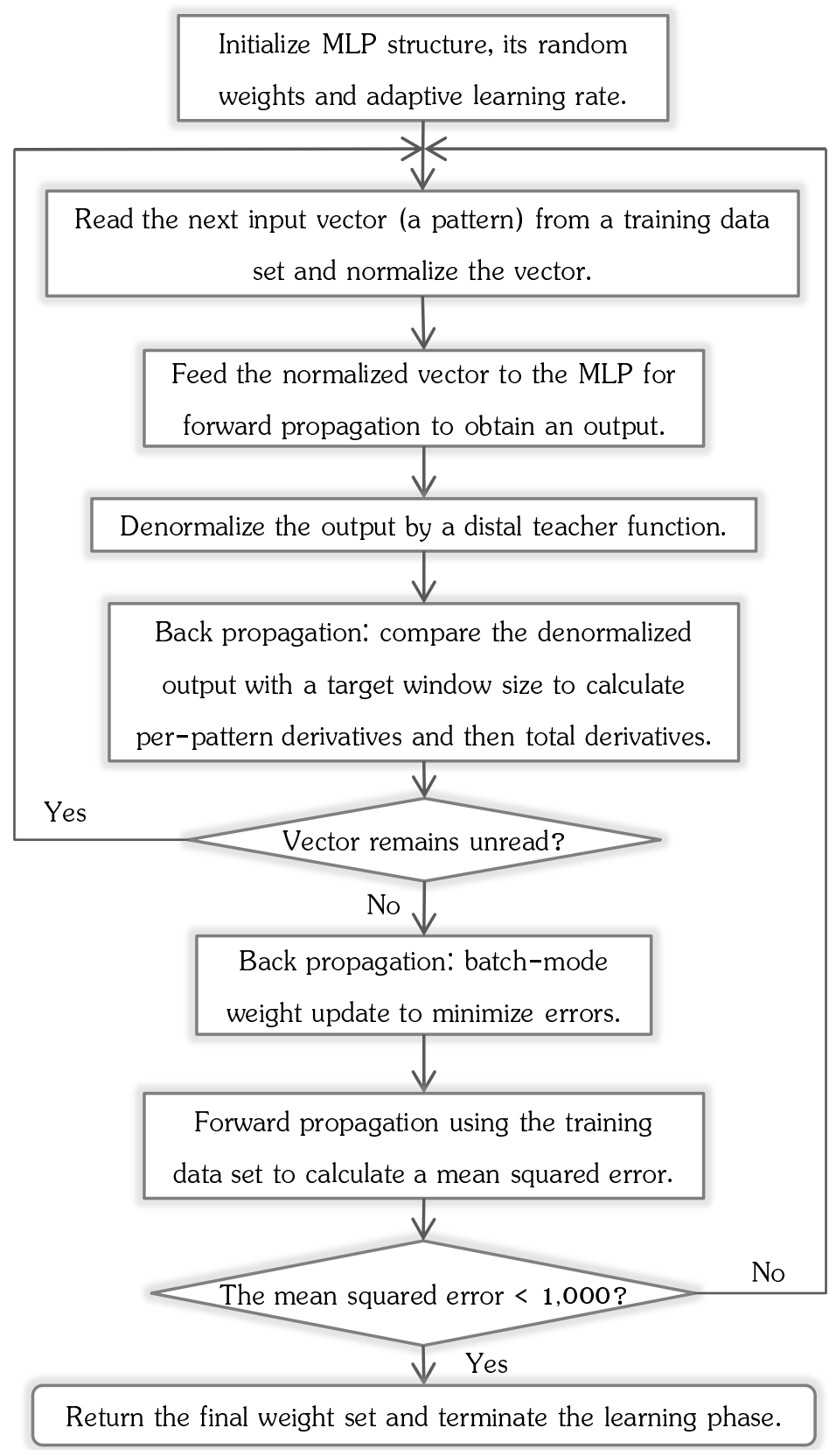

Figure 4: MLP learning phase. 
Table 6: Forecaster training parameters.

\begin{tabular}{ll}
\hline Parameter & Value \\
\hline MLP structure & $10,002 / 2 / 2 / 1$, fully connected \\
Inputs & $\mathrm{X}(\mathrm{Eq} \cdot(6))$ \\
Normalized input range & {$[-1,1]$} \\
Activation function & Sigmoid \\
Objective function & Mean squared error \\
Output & low-level window size \\
Denormalized output range & {$[0,10000]$} \\
Weight update algorithm & Batch-mode back-propagation \\
\hline
\end{tabular}

a batch-mode weight update algorithm. In a batch mode, each pattern $p$ is evaluated to obtain the per-pattern derivative of a single-pattern error with respect to the weights: $\partial E_{p} / \partial w_{i j}=\delta_{j} y_{j}$ where $y_{j}$ is the output activation of a node $j$, and $\delta_{i}$ is the derivative of an error with respect to the weighted sum of node $i$ and given by either $-\left(t_{p}-y_{p}\right) f^{\prime}$ for an output node (where $t_{p}$ and $y_{p}$ are, respectively, target and actual outputs on the $p^{\text {th }}$ pattern, and a sigmoid derivative $f^{\prime}=y(1-y) ; y$ is an MLP output activation) or $f_{i}^{\prime} \sum_{j} w_{j i} \delta_{j}$ for hidden nodes (where $f_{i}^{\prime}=y_{i}\left(1-y_{i}\right) ; y_{i}$ is the output activation of node $i$ ). All per-pattern derivatives are then summed to obtain a total derivative $\partial E / \partial w=\sum_{p} \partial E_{p} / \partial w$ and only then are the weights updated. We used adaptive learning rates instead of fixed ones to make the MLP converge quickly.

During the learning phase, to measure how well the MLP learned and guided the searches for solutions, we used a standard objective function, mean squared error $E_{M S E}=\sum_{p}\left(t_{p}-y_{p}\right)^{2} / P$ where $P$ is the number of training patterns, and $p$ indexes patterns in a training data set. A condition for learning termination was when $E_{M S E}$ was below 1,000 since most MLPs had stabilized after considerably long training times when their $E_{M S E}$ values passed this threshold. Table 6 summarizes the training related parameters of the forecaster module. We have trained totally two MLPs according to the training data sets in Table 5 .

\subsubsection{Validation phase configuration}

Our previous work [36] has shown that measuring the accuracy of each trained MLP (or the forecaster) by using validation data sets to measure errors produced by the forecaster was almost meaningless as a local-optimum window size could have the high degree of validation error. Therefore, instead of validating the forecaster module individually, we have conducted the 
Table 7: Forecaster algorithm

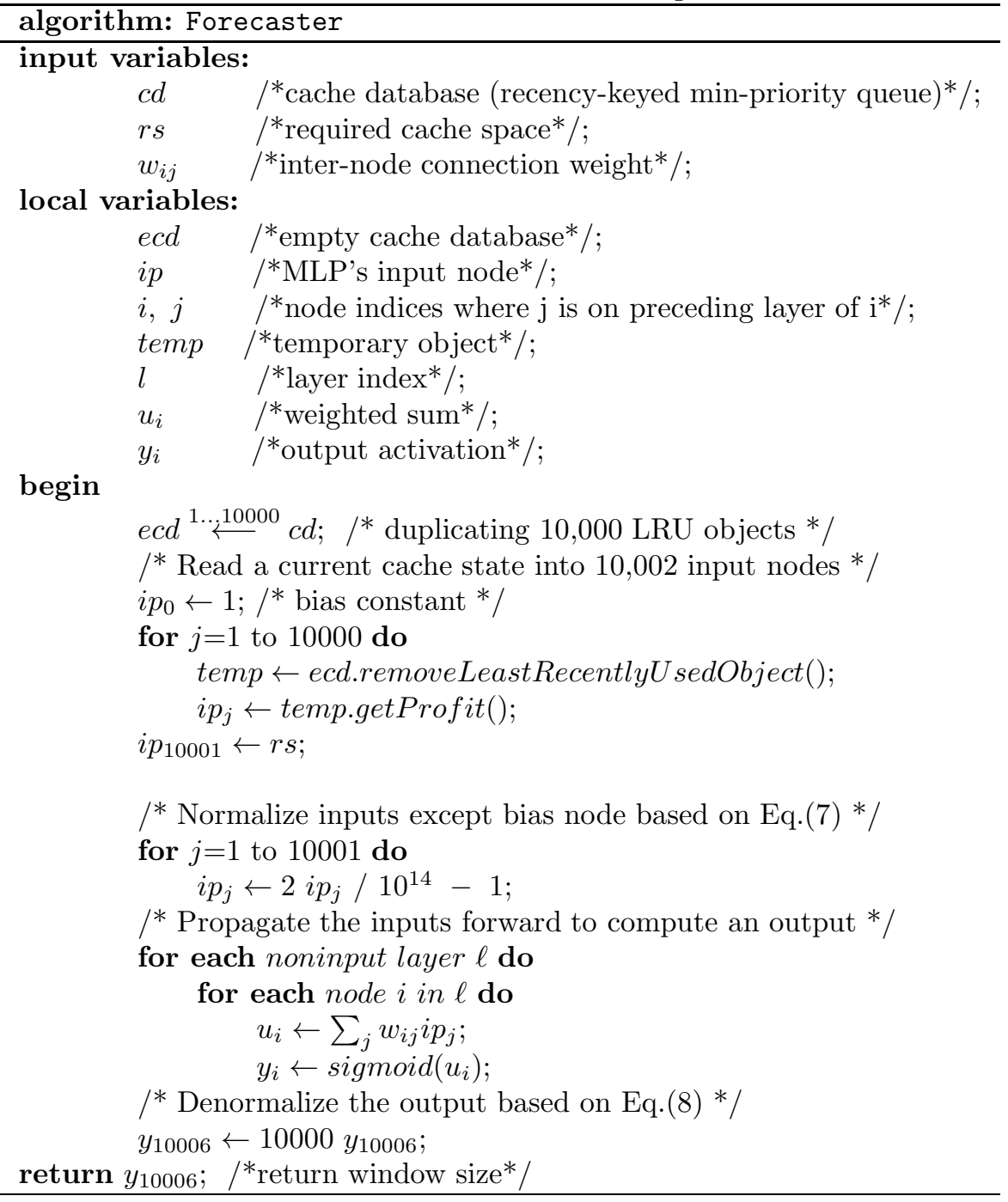

holistic evaluation of i-Cloud that integrated both the forecaster module and Cloud cache replacement policy module as a whole in the four performance metrics based on a trace-driven simulation approach described in Section 5.

Each trained MLP inside the forecaster functions according to the algorithm in Table 7 . It mainly comprises three code portions corresponding to the three components constituting the forecaster module in Fig. 2 (i.e., input vector normalization, MLP and distal teacher). The algorithm's returned 
value, window size, is passed as the input of Cloud algorithm in Table 3.

\section{Results and Discussions}

We have evaluated the i-Cloud by HTTP trace-driven simulations. In particular, the one-month data sets listed in Table 2 have been used as separate workloads to supply the values of i-Cloud parameters denoted in Eq.(5) except $c_{i}$, which was set to either $0.1535 \mathrm{USD} / \mathrm{GB}$ or $0.0829 \mathrm{USD} / \mathrm{GB}$ depending on the domain names of object references as explained in Section 3. Besides using the multiple independent workloads for cross checking, we have also varied the configurations of i-Cloud's window size parameter for comparison purpose as follows.

- Configuration 1: i-Cloud engaged the forecaster, learning BO15D10\% data set in Table 5, to obtain dynamic window sizes intelligently.

- Configuration 2: i-Cloud engaged the forecaster, learning NY15D10\% data set in Table 5 that represents the other different user community's behavior.

- Configuration 3: Nonintelligent-mode i-Cloud where the forecaster module was disabled while Cloud cache replacement policy module used the static optimal window sizes (i.e., $w s_{o}$ in Table 4 according to simulated data sets and cache sizes) to demonstrate the performance upper bounds of i-Cloud.

- Configuration 4: Nonintelligent-mode i-Cloud used the static worst window sizes (i.e., $w s_{w}$ in Table 4 ) to demonstrate i-Cloud's performance lower bounds.

\subsection{Performance evaluation}

Fig. 5 and 6 demonstrate the simulation results of i-Cloud compared with three well-known policies supported by popular Squid caching proxy [30], i.e. LRU, GDSF, and LFU-DA [23], in four performance metrics based on the one-month BO workload and the one-month NY workload presented in Table 2 , respectively. The simulated cache sizes are dictated in percents of the maximum bytes of total unique objects of each of the one-month data sets. i-Cloud(BO15D10\%) and i-Cloud(NY15D10\%) notate the configuration 1 and i-Cloud using the configuration 2, respectively. Infinite cache size, 

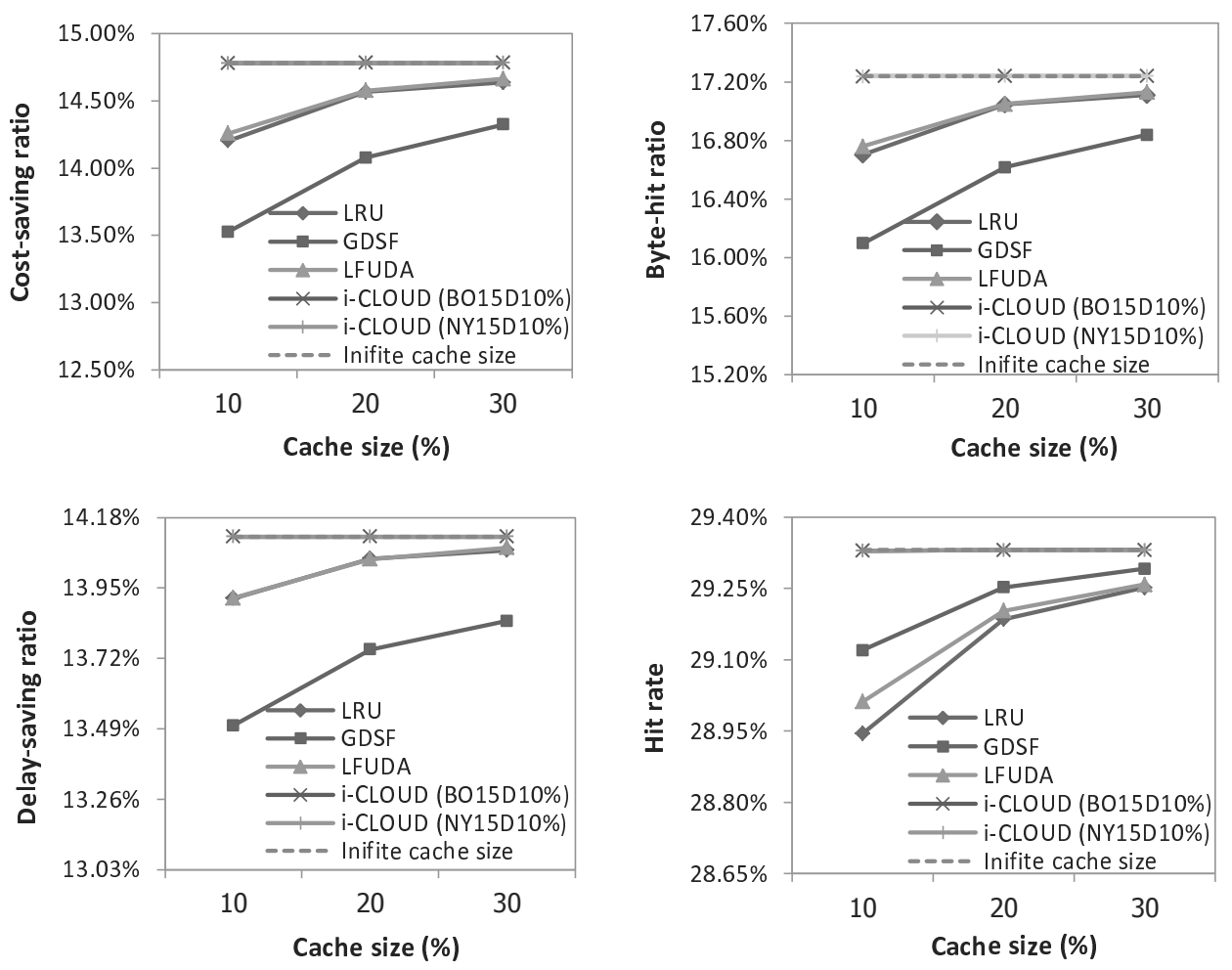

Figure 5: Comparative cost-saving, byte-hit, delay-saving and hit ratios, respectively, of i-Cloud based on 31-day BO data set.

which obviates cache replacement, was also simulated to show ideal caching performances. Each infinite cache size for each data set was set to at least the maximum bytes of total unique objects of the data set given in Table 2 . Interesting observations are as follows.

- For both data sets, it is obvious that i-Cloud has outperformed all competitive policies across all performance metrics. In particular, iCloud(BO15D10\%) and i-Cloud(NY15D10\%) have delivered identically the maximum cost-saving ratio $(14.78 \%)$ and the maximum byte-hit ratio (17.24\%) based on the $\mathrm{BO}$ data set at $30 \%$ cache size, while i-Cloud(NY15D10\%) has delivered the maximum delay-saving ratio (17.96\%) against the NY data set at 30\% cache size. These results indicate one finding that i-Cloud, learning nonuniform cost patterns, has been most efficient for serving the nonuniform cost workloads. The 

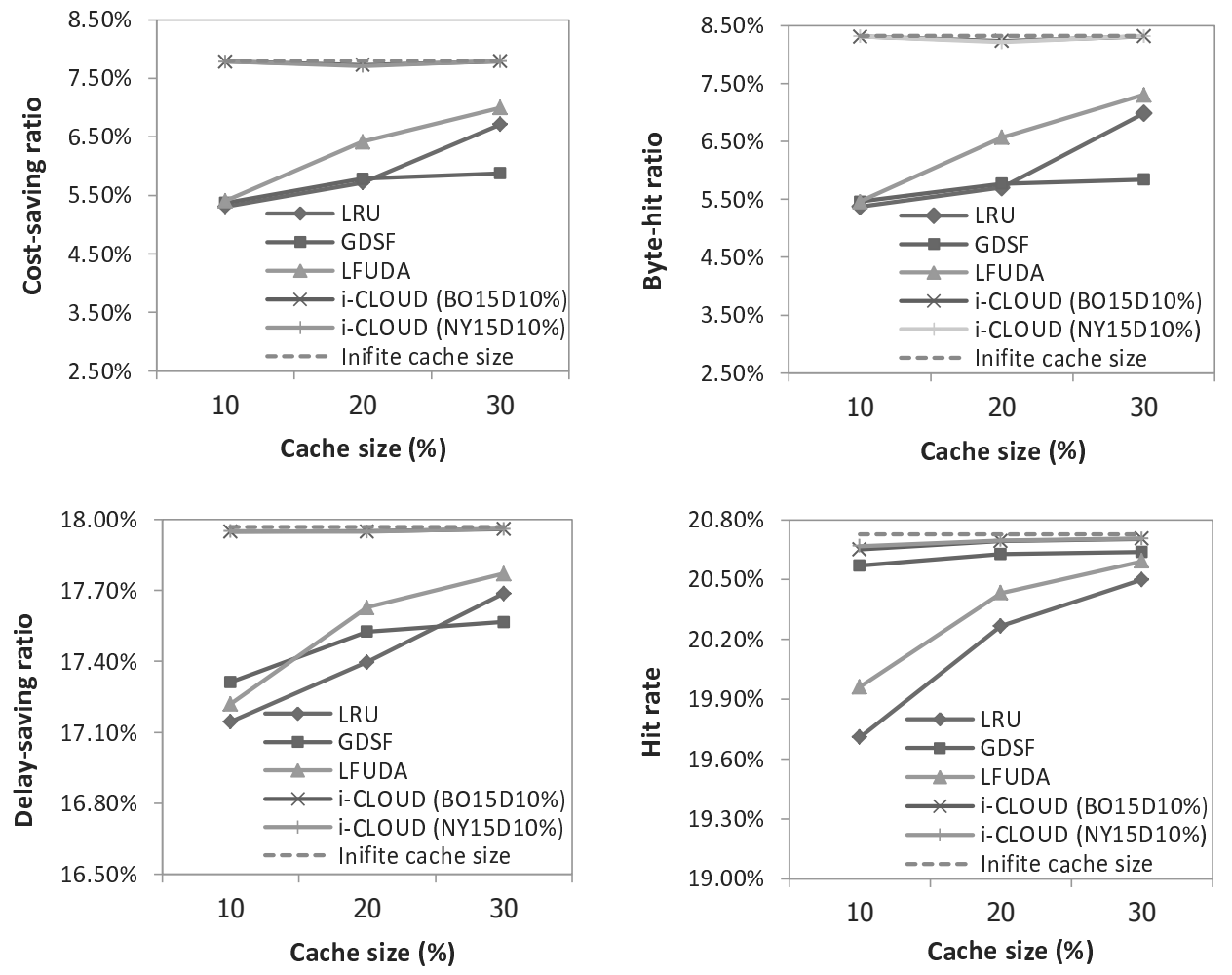

Figure 6: Comparative cost-saving, byte-hit, delay-saving and hit ratios, respectively, of i-Cloud based on 31-day NY data set.

significances of the $14.78 \%$ economical performance, $17.24 \%$ byte-hit performance and $17.96 \%$ delay-saving performance can be interpreted meaningfully based on the representative scenario described in Section 2 as the saved expense of 6,596.41 USD per month, the saved network bandwidth of $65.66 \mathrm{~TB}$ per month and the saved data transfer time of 31.13 hours per month, respectively.

- In terms of cost-saving performance based on the BO data set, iCloud(BO15D10\%) has slightly overcome i-Cloud(NY15D10\%) at 10\% cache size and performed identically to i-Cloud(NY15D10\%) at 20\% and $30 \%$ cache sizes. Using the NY data set, i-Cloud(NY15D10\%) has more economized than i-Cloud (BO15D10\%) at 10\% and 30\% cache sizes and less economized at $20 \%$ cache size. These advocate a finding that i-Cloud performed economically better against user com- 
munity behavior i-Cloud had learned. When comparing byte-hit ratios based on the BO workload, i-Cloud(BO15D10\%) has overcome iCloud(NY15D10\%) at 10\% cache size and been indistinguishable from i-Cloud(NY15D10\%) at 20\% and 30\% cache sizes. With the NY workload, i-Cloud(NY15D10\%) has saved more bandwidth than i-Cloud (BO15D10\%) at $10 \%$ and $30 \%$ cache sizes and less at $20 \%$ cache size. These results support another finding that i-Cloud reduced more network bandwidth against user community behavior i-Cloud had learned. In terms of comparative delay-saving ratios using the BO data set, iCloud(BO15D10\%) has slightly overcome i-Cloud(NY15D10\%) at 10\% cache size and performed the same as i-Cloud(NY15D10\%) at 20\% and $30 \%$ cache sizes. Based on the NY data set, i-Cloud(NY15D10\%) has reduced more transfer time than i-Cloud (BO15D10\%) at 10\% and 30\% cache sizes and less at $20 \%$ cache size. These indicate a finding that i-Cloud saved more transfer time against user community behavior iCloud had learned.

- Across different cache sizes, i-Cloud performances in all kinds of metrics have shown to stabilize at the degrees close to those of infinite cache size. This can be translated as a finding that i-Cloud performances have tolerated the cache sizes varying within the studied range (i.e., $10 \%$ to $30 \%)$.

- Since both i-Cloud(BO15D10\%) and i-Cloud(NY15D10\%) learned short 15-day data sets and have been able to perform well against the one month workloads of both user communities. This substantiates the nondegradation property of i-Cloud's for long-term deployment up to some extent.

- i-Cloud could retain both high byte-hit and hit rates simultaneously against the simulated workloads. Hence, the breakthrough finding in our prior works [28] using the uniform cost model that optimal hit and byte-hit ratios could be attained at the same time also applies to the nonuniform cost environments.

\subsection{Success factor analysis}

This section presents the analysis of success factors to the attained performances of i-Cloud's. The analysis results can be used as a guideline for i-Cloud's performance tuning and as a basis for further research. We first 
show the contributions of window size parameter (i.e., ws in Table 3) followed by those of object's monetary cost per size or data-out charge rate parameter (i.e., $c_{i}$ in Eq.(5)).

The significance of the value selection of window size parameter has been revealed by measuring gaps between the optimal window size-based performances and the worst window size-based performances as shown in Fig. 7: CLOUD(maxima) and CLOUD(minima) denote i-Cloud's performances based on the configurations 3 and 4 , respectively. Interesting observations are as follows.

- The largest effects of window sizes on cost-saving, byte-hit, delay-saving and hit ratios lied totally in the NY workload that are, respectively, $0.60 \%$ at $20 \%$ cache size, $0.78 \%$ at $20 \%$ cache size, $0.10 \%$ at $20 \%$ cache size and $0.07 \%$ at $10 \%$ cache size. These numerical impacts can be interpreted based on the representative scenario as follows: the $0.60 \%$ cost saving, the $0.78 \%$ byte-hit and the $0.10 \%$ delay-saving are eqivalent to $267.78 \mathrm{USD} /$ year, $35.65 \mathrm{~TB} /$ year and 2.08 hours/year, respectively.

- By examining all of the graphs, the window sizes tended to have small effects on all performance aspects when the relative cache sizes were large (i.e., 30\%). This was probably because i-Cloud was executed fewer times when using large cache sizes. Using 10\% and 20\% cache sizes, the performance effects of window sizes have seemed unpredictable.

To reveal the benefits gained from being aware of the difference of dataout charge rates in the object profit calculation (Eq.(5)), we conducted the performance measurement of the configuration 3 for $2 \%, 5 \%, 10 \%, 20 \%$ and $30 \%$ cache sizes and compared results with those generated by the same configuration except treating all data-out charge rates equally for all objects in object profit calculations (i.e., $c_{i}$ in Eq.(5) was always set to a single assumed constant). Basically, the results of both configurations were so closed to each other that it was hard to discern gaps between graph lines if they were plotted into trend charts as the former figure. Interesting observations are described as follows.

- Among all simulated workloads and cache sizes, ignoring the differences among data-out charge rates had caused the most degradations of costsaving, byte-hit, delay-saving and hit performances by $0.042 \%, 0.017 \%$, $0.032 \%$ and $0.21 \%$ at $2 \%$ cache size when using the BO workload. The 

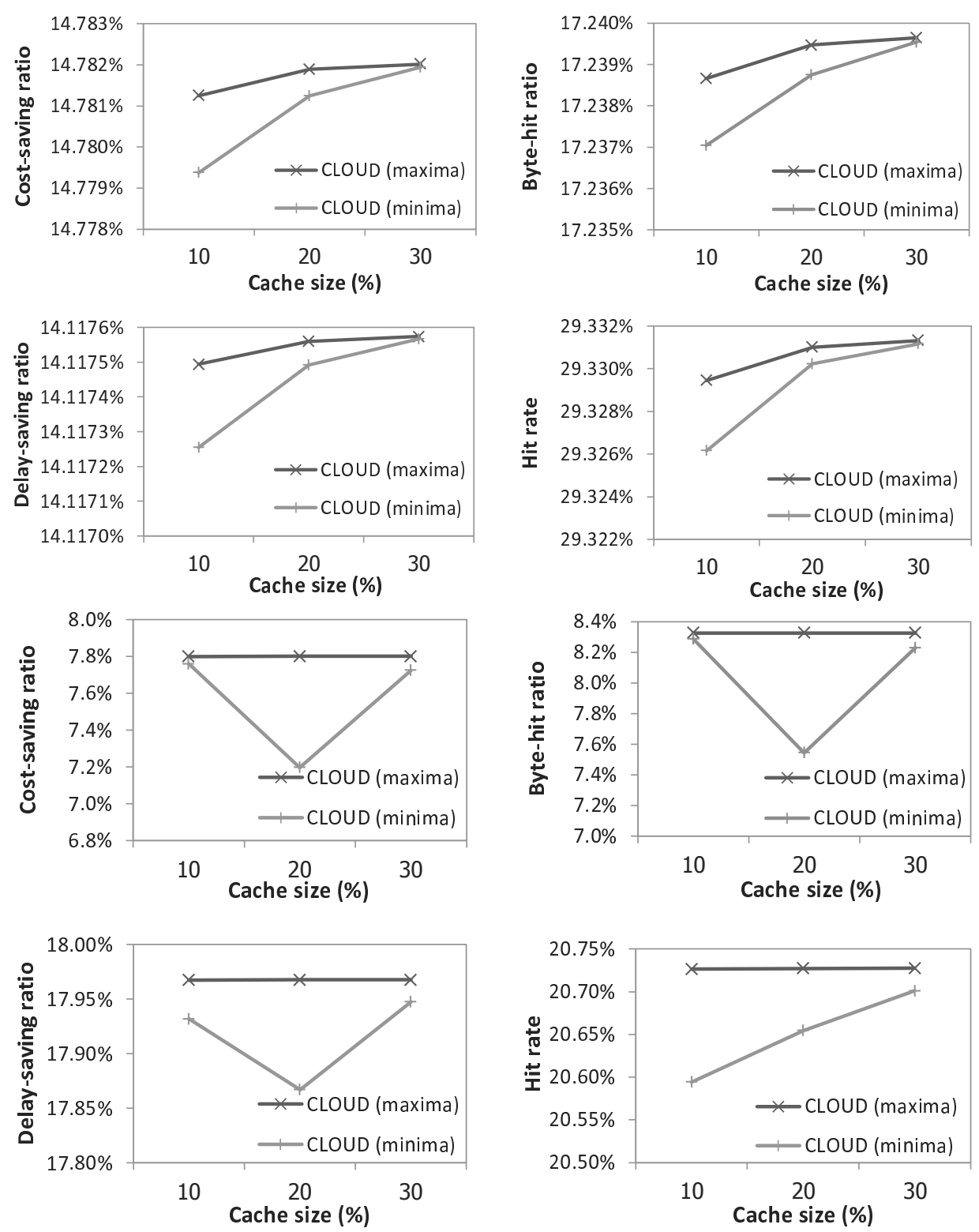

Figure 7: Effects of window size variation to cost-saving, byte-hit, delay-saving and hit ratios, respectively, based on the 31-day BO data set (two upper rows) and 31-day NY data set (two lower rows).

significances of these effects can be interpreted based on the representative scenario as follows: the $0.042 \%$ cost saving, the $0.017 \%$ byte-hit and 
the $0.032 \%$ delay-saving are equivalent to $225 \mathrm{USD} /$ year, $796 \mathrm{~GB} /$ year and 40 minutes/year, respectively. Although these amounts seem to be trivial, they might gain more significances if the gap between dataout charge rates became wider (e.g., when accessing both an internal private cloud without any charge and an external public cloud with charge) and the data transferred out of both cloud providers became unbalanced. (Recall that in Sect. 2, we assumed that the amounts of data transfers egress from two public clouds were balanced.)

- It was found that accounting the objects' actual data-out charge rates into cache replacement decisions has tended to contribute more in all performance metrics when the relative cache size has decreased. This was possibly because i-Cloud (and the $c_{i}$ ) was executed more frequently when deploying smaller cache sizes.

\section{Related Works}

We have characterized a number of client-side web cache eviction schemes based on their key parameters that distinguish them from our scheme as follows.

\subsection{Object size, downloading monetary cost and access frequency}

A number of policies surveyed in [23]: LRU, LFU-DA, EXP1, Value-Aging, HLRU, LFU, LFU-Aging, $\alpha$-Aging, swLFU, SLRU, Generational Replacement, LRU*, LRU-Hot, Server assisted cache replacement, LR, RAND, LRU$\mathrm{C}$, Randomized replacement with general value functions, including policies ARC [37], CSOPT [38], LA2U [39], LAUD [39], SEMALRU [40] and LRUSLFR [41] do not parameterize object sizes. If big objects are requested frequently but often evicted by these policies (as blind to object sizes), caching proxy will have to reload the big objects from their original servers frequently as well. As a result, object-size ignoring schemes permit the poor economies of data-out charges and poor cloud scalability.

Another group of policies surveyed in [23]: GDSF, LRU-Threshold, LRUMin, SIZE, LOG2-SIZE, PSS, LRU-LSC, Partitioned Caching, HYPERG, CSS, LRU-SP, GD-Size, GD*, TSP, MIX, HYBRID, LNC-R-W3, LUV, HARMONIC, LAT, GDSP, LRU-S, including LNC-R-W3-U [42], SE [43], R-LPV [44], Min-SAUD [45], OPT [46], LPPB-R [47], OA [48], CSP [49], GA-Based Cache Replacement Policy [50], improved GD* [51] and SzLFU(k) 
[52] consider object sizes in such a way that replacing big objects first, thus neither aiming for cloud scalability nor economy.

The other policies LRV [23], M-Metric [23] and Bolot and Hoschkas [53] favor big objects just like i-Cloud. In particular, LRV supports the factorization of object size and either downloading latency or downloading monetary cost; M-Metric allows big objects to stay longer in cache but does not take downloading monetary cost into account; Bolot and Hoschka's policy replaces smaller objects first regardless of downloading monetary cost and spatial locality (i.e., access frequency).

\subsection{Access recency}

All known policies prioritize the recencies of object requests either implicitly or both implicitly and explicitly. By implicitly, every policy always accepts a newly loaded missing object (i.e., the most recently used object) into cache rather than rejects it. By explicitly, several policies such as LRU, LRU-Threshold, SIZE, LRU-Min, EXP1, Value-Aging, HLRU, PSS, LRULSC, Partitioned Caching, SLRU, Generational Replacement, LRU*, LRUHot, HYPER-G, CSS and LRU-SP parameterize elapsed times since the last

requests to objects. i-Cloud policy regards the recency property of objects in its model in both implicit and explicit manner.

\subsection{Object downloading latency}

Several policies: GD-Size, GDSF, GD*, improved GD*, GDSP, HYBRID, LAT, LUV, MIX, LNC-R-W3, LNC-R-W3-U and LRU-SLFR take object downloading latencies into account. All of them replace objects having shorter downloading latencies first. i-Cloud also follows this approach.

\subsection{Object expiration}

Few policies consider object expiration. LA2U, LAUD, improved GD* and LNC-R-W3-U replace frequently updated objects first. The former three, however, do not describe how update frequencies are derived. The last policy estimates update frequencies from changes detected in HTTP's Last-Modified header fields; however, if frequently updated objects are seldom requested, updated Last-Modified values will be rarely perceived by the policy resulting in underestimated update frequencies. This problem can be solved by using explicit expiration time or time-to-live (TTL) parameter as in Bolot and Hoschka's policy even though the parameter had not yet been implemented in their presented empirical studies. 


\subsection{Object cacheability}

NNPCR [54] and its extension, NNPCR-2 [55], apply artificial neural networks to rate object cacheability. The networks were supervised to learn cacheable patterns from valid HTTP status codes and object sizes. The perceptrons' inputs are access frequencies, recencies and object sizes while outputs are rating scores. A lower rating score represents a better choice for replacement. Intelligent long-term cache removal algorithm based on adaptive neuro-fuzzy inference system (ANFIS) [56] takes access frequency, recency, object size and downloading latency as the inputs of a trained ANFIS to dictate noncacheable objects. In ANFIS training, objects requested again at later point in specific time are considered cacheable. The oldest noncacheable object is removed first. Intelligent web caching using artificial neural network and particle swarm optimization algorithm [57] trained the network to keep slowly downloading, big and frequently accessed objects in cache. Adaptive web cache predictor [58] utilizes artificial neural network and sliding windows to find whether objects will be re-accessed for at least certain times within the particular number of following accesses. In training phase, the network learns type of object, number of previous hits, relative access frequency, object size and downloading latency. Training targets are the most likely re-accessed objects that are the objects reaccessed at least certain times within forward-looking window. Reference [59] applies logistic function to compute re-access probability based on an object size, object type (e.g., text, image, video, executable), access frequency and recency. In learning phase, objects re-accessed at least once in next certain accesses obtain high probabilities, while an object with the lowest probability is replaced first. LRU-M [60] utilizes a multinominal logistic regression model to classify objects into multilevel classes for classful LRU replacement. The model was trained by using an access frequency, recency, delay, object size, type and popularity consistency. All of these algorithms, however, omit both downloading monetary cost and object expiration factors that are useful for controlling nonuniform data-out charge rates and cache pollution due to stale objects, respectively. SVM-GDSF, C4.5-GDS and SVM-LRU [61] incorporate support vector machines (SVM-) and a decision tree (C4.5-) with conventional policies GDSF, GD-Size and LRU, respectively. The support vector machines add the information of object revisiting classification into the base policies. The decision tree gives the information of object revisiting probability. These variant policies still suffer the shortcomings of their base policies described earlier. A web cache replacement policy with nonlinear 
model using object features [62] modifies LRV by integrating an artificial neural network. The network was trained with feature vectors comprising the numbers of certain HTML tags and HTTP log attributes (e.g., document size, expiration and content type). The network's output, re-access probability, together with cost (e.g., object downloading latency or object size) and the benefit of purging the object from cache (proportional to freed cache space) are used to produce the object's relative value. The policy replaces an object with the lowest relative value first. The policy also inherits the aforementioned drawback of LRV and totally ignores both access frequency and recency.

To recap up to this point, no explicit policy aims for cloud computing paradigm for at least one of the following main reasons. First, those policies evict big objects to optimize hit rates rather than the scalability of cloud transport infrastructures and the responsiveness of cloud computing services. Second, they are not optimized for varying public cloud data-out charges, thus neither improve cloud consumer-side cloud economy nor support the efficient uses of multi-provider clouds.

Reference [29] has originally proposed Cloud strategy as an early attempt of client-side cloud cache replacement policy. Its performances were investigated by using short 5-day data sets. A finding in such work was invalidated by our subsequent work [63], in which we have made the minor Cloud algorithm enhancement and conducted performance investigation by using additionally 15 day data sets resulting in a groundbreaking finding in the realm of heterogeneous multicomputer caching that is optimal byte-hit and hit performances can actually be achieved at the same time. The early investigations of the nonuniform cost models could also be found in both of these works. In [28], a new set of even longer one-month data sets was used to emulate more realistic environments to ensure Cloud's performance behaviors and the finding of [63]. We have also studied Cloud policy in fully nonuniform cost circumstances against one-month data sets in [25]. Reference [36] has originally improved Cloud policy by enlisting an artificial neural network (ANN). A result is i-Cloud cache replacement policy, which can adapt itself intelligently to evolving workload to maintain superior performances. However, such work has been studied totally in uniform cost environments.

As the novel contribution of this paper, we have presented the new learning configurations, performance behaviors and findings of i-Cloud completely trained and deployed in multi-provider cloud or nonuniform cost environments. The results have also been compared with LRU, GDSF and LFUDA, 
the well-known strategies supported by popular Squid caching proxy. In addition, we have also conducted the analysis of i-Cloud's success factors to guide appropriate performance tuning as well as future research. On the other hand, the merit of this paper is a well-proven approach to address cloud economy and efficiency for consumer organizations utilizing multiple cloud providers.

\section{Conclusions}

This paper presents i-Cloud cache replacement policy that is capable of improving multi-provider cloud economy, scalability and responsiveness. iCloud has access recency as a priority factor for object replacement decision. i-Cloud parameterizes an MLP-based self-tuning window size to generalize the recencies of objects within a formulated object cluster. The lowest profitable clustered objects are purged from cloud cache. By trace-driven simulations, we discovered the following facts. i-Cloud outperformed the well-known strategies, LRU, GDSF and LFU-DA, in all nonuniform cost environments. i-Cloud tended to perform well not only in its learned environments but also for longer run than its training durations. As a breakthrough finding, i-Cloud could attain both high byte-hit and hit rates at the same time based on our workloads. i-Cloud could deliver stable optimal performances in byte-hit, cost-saving and delay-saving ratio metrics based on most simulated cache sizes. Tuning the window sizes tended to have small effects in all performance metrics when the relative cache sizes were large. Finally, considering the data-out charge rate differences during cache replacement decision makings has tended to contribute more in all performance improvements when the relative cache size has decreased.

\section{Acknowledgments}

This research is financially supported by Thailand's Office of the Higher Education Commission, Thailand Research Fund, and Sripatum university (grant MRG5580114). The authors also thank Duane Wessels, National Science Foundation (grants NCR-9616602 and NCR-9521745) and the National Laboratory for Applied Network Research for the trace data used in this study. 


\section{References}

[1] Amazon.com, Inc., Amazon Web Services: Big Data, 1 February 2014. URL: http://aws . amazon. com/big-data/.

[2] Google Inc., Google App Engine: BigQuery, 1 February 2014. URL: http://cloud.google.com/products/bigquery.

[3] Microsoft, Windows Azure: Big Data, 1 February 2014. URL: http://www.windowsazure.com/en-us/solutions/big-data/.

[4] Amazon.com, Inc., Amazon Web Services, 1 December 2013. URL: http://aws . amazon.com/s3/.

[5] Google Inc., Google App Engine, 1 December 2013. URL: http://cloud.google.com/.

[6] Microsoft, Windows Azure, 1 December 2013. URL: http: //www.windowsazure.com/.

[7] M. Armbrust, A. Fox, R. Griffith, A. D. Joseph, R. H. Katz, A. Konwinski, G. Lee, D. A. Patterson, A. Rabkin, I. Stoica, M. Zaharia, Above the Clouds: A Berkeley View of Cloud Computing, Technical Report UCB/EECS-2009-28, EECS Department, University of California, Berkeley, 2009.

[8] M. Armbrust, A. Fox, R. Griffith, A. D. Joseph, R. Katz, A. Konwinski, G. Lee, D. Patterson, A. Rabkin, I. Stoica, M. Zaharia, A view of cloud computing, Commun. ACM 53 (2010) 50-58.

[9] S. Marston, Z. Li, S. Bandyopadhyay, J. Zhang, A. Ghalsasi, Cloud computing the business perspective, Decision Support Systems 51 (2011) $176-189$.

[10] C. Momm, R. Krebs, A qualitative discussion of different approaches for implementing multi-tenant saas offerings., in: R. Reussner, A. Pretschner, S. Jhnichen (Eds.), Software Engineering (Workshops), volume 184 of LNI, GI, 2011, pp. 139-150.

[11] Amazon.com, Inc., Amazon CloudFront, 1 December 2013. URL: http://aws . amazon. com/cloudfront/. 
[12] C. Sriwiroj, T. Banditwattanawong, A cost model for client-side caas, in: K. J. Kim (Ed.), Information Science and Applications, volume 339 of Lecture Notes in Electrical Engineering, Springer Berlin Heidelberg, 2015, pp. 361-368.

[13] D. Petcu, Multi-cloud: Expectations and current approaches, in: Proceedings of the 2013 International Workshop on Multi-cloud Applications and Federated Clouds, MultiCloud '13, ACM, New York, NY, USA, 2013, pp. 16. URL: http://doi.acm.org/10.1145/2462326.2462328. doi:10.1145/2462326.2462328.

[14] P. Wright, T. Harmer, J. Hawkins, Y. L. Sun, A commodity-focused multi-cloud marketplace exemplar application, in: Cloud Computing (CLOUD), 2011 IEEE International Conference on, 2011, pp. 590-597.

[15] P. M. Mell, T. Grance, SP 800-145. The NIST Definition of Cloud Computing, Technical Report, Gaithersburg, MD, United States, 2011.

[16] K. Bernsmed, M. Jaatun, P. Meland, A. Undheim, Thunder in the clouds: Security challenges and solutions for federated clouds, in: Cloud Computing Technology and Science (CloudCom), 2012 IEEE 4th International Conference on, 2012, pp. 113-120.

[17] S. Bardhan, D. Milojicic, A mechanism to measure quality-of-service in a federated cloud environment, in: Proceedings of the 2012 Workshop on Cloud Services, Federation, and the 8th Open Cirrus Summit, FederatedClouds '12, 2012, pp. 19-24.

[18] D. Yuan, X. Liu, L. Cui, T. Zhang, W. Li, D. Cao, Y. Yang, An algorithm for cost-effectively storing scientific datasets with multiple service providers in the cloud, in: eScience (eScience), 2013 IEEE 9th International Conference on, 2013, pp. 285-292.

[19] T. Genez, L. Bittencourt, E. Madeira, Using time discretization to schedule scientific workflows in multiple cloud providers, in: Cloud Computing (CLOUD), 2013 IEEE Sixth International Conference on, 2013, pp. 123-130. 
[20] T. Ermakova, B. Fabian, Secret sharing for health data in multi-provider clouds, in: Business Informatics (CBI), 2013 IEEE 15th Conference on, 2013, pp. 93-100.

[21] M. AlZain, E. Pardede, B. Soh, J. Thom, Cloud computing security: From single to multi-clouds, in: System Science (HICSS), 2012 45th Hawaii International Conference on, 2012, pp. 5490-5499.

[22] S. Liu, X. Huang, H. Fu, G. Yang, Understanding data characteristics and access patterns in a cloud storage system, in: Cluster, Cloud and Grid Computing (CCGrid), 2013 13th IEEE/ACM International Symposium on, 2013, pp. 327-334.

[23] S. Podlipnig, L. Böszörmenyi, A survey of web cache replacement strategies, ACM Comput. Surv. 35 (2003) 374-398.

[24] A. Balamash, M. Krunz, An overview of web caching replacement algorithms, Communications Surveys Tutorials, IEEE 6 (2004) 44-56.

[25] T. Banditwattanawong, P. Uthayopas, Cloud-enabling technique for dissimilar charges, in: The 2014 International Industrial Information Systems Conference (IIISC2014), 2014, pp. 119-120.

[26] National Laboratory for Applied Network Research, Weekly squid http access logs, 2012. URL: http://www .ircache.net/.

[27] F. Douglis, A. Feldmann, B. Krishnamurthy, J. Mogul, Rate of change and other metrics: a live study of the world wide web, in: Proceedings of the USENIX Symposium on Internet Technologies and Systems, USITS'97, USENIX Association, Berkeley, CA, USA, 1997.

[28] T. Banditwattanawong, P. Uthayopas, A client-side cloud cache replacement policy, ECTI Transactions on Computer and Information Technology (2014) in press.

[29] T. Banditwattanawong, From web cache to cloud cache, in: R. Li, J. Cao, J. Bourgeois (Eds.), Advances in Grid and Pervasive Computing, volume 7296 of Lecture Notes in Computer Science, Springer Berlin / Heidelberg, 2012, pp. 1-15.

[30] D. Wessels, Squid : the definitive guide, O Reilly, 2004. 
[31] R. Fielding, J. Gettys, J. Mogul, H. Frystyk, L. Masinter, P. Leach, T. Berners-Lee, Hypertext transfer protocol http/1.1, 2012. URL: http://www. ietf.org/rfc/rfc2616.txt.

[32] R. D. Reed, R. J. Marks, Neural Smithing: Supervised Learning in Feedforward Artificial Neural Networks, MIT Press, Cambridge, MA, USA, 1998.

[33] M. Arlitt, R. Friedrich, T. Jin, Performance evaluation of web proxy cache replacement policies, Perform. Eval. 39 (2000) 149-164.

[34] M. I. Jordan, D. E. Rumelhart, Forward models: Supervised learning with a distal teacher, Cognitive Science 16 (1992) 307-354.

[35] J. Sola, J. Sevilla, Importance of input data normalization for the application of neural networks to complex industrial problems, Nuclear Science, IEEE Transactions on 44 (1997) 1464-1468.

[36] T. Banditwattanawong, P. Uthayopas, An intelligent cloud cache replacement scheme, in: B. Papasratorn, N. Charoenkitkarn, V. Vanijja, V. Chongsuphajaisiddhi (Eds.), Advances in Information Technology, volume 409 of Communications in Computer and Information Science, Springer, 2013, pp. 23-34.

[37] N. Megiddo, D. Modha, Outperforming lru with an adaptive replacement cache algorithm, Computer 37 (2004) 58-65.

[38] J. Jeong, M. Dubois, Cache replacement algorithms with nonuniform miss costs, Computers, IEEE Transactions on 55 (2006) 353-365.

[39] H. Chen, Y. Xiao, X. Shen, Update-based cache access and replacement in wireless data access, Mobile Computing, IEEE Transactions on 5 (2006) 1734-1748.

[40] K. Geetha, N. Ammasai Gounden, S. Monikandan, Semalru: An implementation of modified web cache replacement algorithm, in: Nature Biologically Inspired Computing, 2009. NaBIC 2009. World Congress on, 2009, pp. 1406-1410.

[41] S. won Shin, K.-Y. Kim, J. su Jang, Lru based small latency first replacement (slfr) algorithm for the proxy cache, in: Web Intelligence, 
2003. WI 2003. Proceedings. IEEE/WIC International Conference on, 2003, pp. 499-502.

[42] J. Shim, P. Scheuermann, R. Vingralek, Proxy cache algorithms: design, implementation, and performance, Knowledge and Data Engineering, IEEE Transactions on 11 (1999) 549-562.

[43] A. R. Sarma, R. Govindarajan, An efficient web cache replacement policy., in: T. M. Pinkston, V. K. Prasanna (Eds.), HiPC, volume 2913 of Lecture Notes in Computer Science, Springer, 2003, pp. 12-22.

[44] N. Chand, R. Joshi, M. Misra, Data profit based cache replacement in mobile environment, in: Wireless and Optical Communications Networks, 2006 IFIP International Conference on, 2006, pp. 5 pp.-5.

[45] J. Xu, Q. Hu, W.-C. Lee, D. Lee, Performance evaluation of an optimal cache replacement policy for wireless data dissemination, Knowledge and Data Engineering, IEEE Transactions on 16 (2004) 125-139.

[46] L. Yin, G. Cao, Y. Cai, A generalized target-driven cache replacement policy for mobile environments, in: Applications and the Internet, 2003. Proceedings. 2003 Symposium on, 2003, pp. 14-21.

[47] K. Kim, D. Park, Least popularity-per-byte replacement algorithm for a proxy cache, in: Parallel and Distributed Systems, 2001. ICPADS 2001. Proceedings. Eighth International Conference on, 2001, pp. 780-787.

[48] K. Li, T. Nanya, W. Qu, A minimal access cost-based multimedia object replacement algorithm, in: Parallel and Distributed Processing Symposium, 2007. IPDPS 2007. IEEE International, 2007, pp. 1-7.

[49] P. Triantafillou, I. Aekaterinidis, Web proxy cache replacement: do's, don'ts, and expectations, in: Network Computing and Applications, 2003. NCA 2003. Second IEEE International Symposium on, 2003, pp. $59-66$.

[50] Y. Chen, Z.-Z. Li, Z.-W. Wang, A ga-based cache replacement policy, in: Machine Learning and Cybernetics, 2004. Proceedings of 2004 International Conference on, volume 1, 2004, pp. 263-266 vol.1. 
[51] K. Li, H. Shen, An improved greedydual cache document replacement algorithm, in: Web Intelligence, 2004. WI 2004. Proceedings. IEEE/WIC/ACM International Conference on, 2004, pp. 457-460.

[52] H. Wang, J. Peng, Y. Wu, H. Feng, Szlfu(k) web cache replacement algorithm, in: TENCON '02. Proceedings. 2002 IEEE Region 10 Conference on Computers, Communications, Control and Power Engineering, volume 2, 2002, pp. 754-758 vol.2.

[53] J.-C. Bolot, P. Hoschka, Performance engineering of the world wide web: application to dimensioning and cache design, Comput. Netw. ISDN Syst. 28 (1996) 1397-1405.

[54] J. Cobb, H. ElAarag, Web proxy cache replacement scheme based on back-propagation neural network, J. Syst. Softw. 81 (2008) 1539-1558.

[55] S. Romano, H. ElAarag, A neural network proxy cache replacement strategy and its implementation in the squid proxy server, Neural Comput. Appl. 20 (2011) 59-78.

[56] W. Ali, S. M. Shamsuddin, Intelligent client-side web caching scheme based on least recently used algorithm and neuro-fuzzy system, in: Proceedings of the 6th International Symposium on Neural Networks: Advances in Neural Networks - Part II, ISNN 2009, Springer-Verlag, Berlin, Heidelberg, 2009, pp. 70-79.

[57] S. Sulaiman, S. Shamsuddin, F. Forkan, A. Abraham, Intelligent web caching using neurocomputing and particle swarm optimization algorithm, in: Modeling Simulation, 2008. AICMS 08. Second Asia International Conference on, 2008, pp. 642-647.

[58] W. Tian, B. Choi, V. V. Phoha, An adaptive web cache access predictor using neural network, in: Proceedings of the 15th international conference on Industrial and engineering applications of artificial intelligence and expert systems: developments in applied artificial intelligence, IEA/AIE '02, Springer-Verlag, London, UK, UK, 2002, pp. 450-459.

[59] A. Foong, Y.-H. Hu, D. Heisey, Logistic regression in an adaptive web cache, Internet Computing, IEEE 3 (1999) 27-36. 
[60] G. P. Sajeev, M. P. Sebastian, A novel content classification scheme for web caches, Evolving Systems 2 (2011) 101-118.

[61] W. Ali, S. M. Shamsuddin, A. S. Ismail, Intelligent web proxy caching approaches based on machine learning techniques, Decis. Support Syst. 53 (2012) 565-579.

[62] T. Koskela, J. Heikkonen, K. Kaski, Web cache optimization with nonlinear model using object features, Computer Network 43 (2003) 805817.

[63] T. Banditwattanawong, P. Uthayopas, Cloud cache replacement policy: new performances and findings, in: Annual PSU Phuket, 2012. PSU PIC 2012. 1st International Conference on, 2013. 